\title{
Magmatic degassing and the volatile budget of the Moon, contributions by Malcolm J. Rutherford
}

\author{
ALberto SAAL ${ }^{1}$, ERIK HAURI ${ }^{2}$, JAMES VAN ORMAN ${ }^{3}$, \\ MALCOLM RUTHERFORD ${ }^{1}$ \\ ${ }^{1}$ DEEPS, Brown University, Providence, RI 02912, USA \\ ${ }^{2}$ DTM, Carnegie Institution of Washington, DC 20015, USA \\ ${ }^{3}$ DEEPS, Case Western Reserve University, Cleveland, $\mathrm{OH}$ \\ 44106, USA
}

Establishing the origin of water and other volatiles, and how and when the budgets of these elements were set in planetary bodies during the earliest stages of Solar System evolution is a fundamental step to elucidate the mechanisms of planetary formation and differentiation. The Moon provides a frozen record of the first few hundred million years of Solar System evolution and, thus, offers a unique opportunity to establish the origin, budget and timing of the water and other volatiles delivery to the terrestrial planets. The experimental and analytical work on highly- and moderately-volatile elements in lunar volcanic glasses and melt inclusions by MJ Rutherford and colleagues has provided the first definitive evidence for the accretion and retention of these elements in the Moon's interior [1,2]. Measurement of $\mathrm{H}$ in lunar apatite, at levels similar to terrestrial apatite, has added weight to this discovery $[3,4]$. These results are at odds with the longest-standing observations that the abundances of highly- and moderatelyvolatile elements in lunar basalts are as much as 1000 times more depleted than in terrestrial basalts [5]. Rutherford and colleagues shown that most of these apparent contradictions have arisen due to the previously unappreciated importance of a single widespread process, magmatic degassing [6,7]. Degassing occurs in all eruptions of magma, with the consequent release of volatile elements into an exsolved vapor phase. We use ours and previously published results to evaluate lunar magmatic degassing and show that volatile element contents for the bulk silicate Moon (BSM) are only moderately depleted compared with the bulk silicate Earth (avg. $0.25 \mathrm{x}$ BSE), overlapping with the composition of the terrestrial depleted MORB source. Before the Moon's extent of volatile depletion can be confidently attributed to the accretion processes, magmatic degassing must be examined and critically evaluated.

References [1] Saal et al., 2008. Nature 454, 192. [2] Hauri et al., 2015. FEPS 409, 252. [3] Boyce et al., 2014. Sc. 344, 400. [4] Anand et al., 2014. PTRS A 372. [5] Albarede et al., 2014. MAPS.12331. [6] Wetzel et al., 2013. PNAS, 110, 8010. [7] Wetzel et al., 2015. Nature Geosc. 8, 755. 